\title{
The "double whammy" of a continuous-flow left ventricular assist device on von Willebrand factor
}

Jay R. Hydren, MS, ${ }^{a}$ Russell S. Richardson, PhD, ${ }^{\mathrm{a}, \mathrm{b}, \mathrm{c}}$ Omar Wever-Pinzon, MD, ${ }^{\mathrm{d}}$ and Stavros G. Drakos, MD, $\mathrm{PhD}^{\mathrm{d}}$

From the ${ }^{\mathrm{a}}$ Department of Nutrition and Integrative Physiology, and Divisions of ${ }^{\mathrm{b}}$ Geriatrics and ${ }^{\mathrm{d}}$ Cardiovascular Medicine, Department of Internal Medicine, University of Utah, Salt Lake City, Utah; and ${ }^{\mathrm{c}}$ Geriatric Research, Education, and Clinical Center, Salt Lake City VA Medical Center, Salt Lake City, Utah.

This research was supported by the National Institutes of Health under Ruth L. Kirschstein National Research Service Award National Institutes of Health 1T322HL139451 from the National Heart, Lung, and Blood Institute.

Received for publication Nov 26, 2018; revisions received March 4, 2019; accepted for publication March 13, 2019; available ahead of print June 28, 2019.

Address for reprints: Stavros G. Drakos, MD, PhD, University of Utah Hospital, Heart Failure and Recovery Clinic, 30 North 1900 East, Suite 4A100, Salt Lake City, UT 84132 (E-mail: Stavros.Drakos@hsc.utah.edu). J Thorac Cardiovasc Surg 2020;159:910-5

0022-5223/\$0.00

Published by Elsevier Inc. on behalf of The American Association for Thoracic Surgery https://doi.org/10.1016/j.jtcvs.2019.03.133

Mounting evidence points to both arterial pulsatility and pump shear as factors that may be responsible for the high incidence of nonsurgical bleeding events observed in patients with heart failure who received a continuous-flow left ventricular assist device (CF-LVAD). Nonsurgical bleeding, particularly gastrointestinal bleeding (GIB), significantly affects the quality of life and survival in CF-LVADsupported patients. ${ }^{1-4}$ As mechanical circulatory support technology has been refined, durability was improved at the expense of arterial pulsatility. That is concerning given that arterial vascular pulsatility co-evolved with closed circulatory loops as multi-cellular organisms developed, it is highly likely that the regulation of clotting factors, specifically von Willegbrand Factor (vWF), will be affected by such unphysiologically low arterial pulsatility. Indeed, a plethora of observational evidence, from our institute and others, points to a potential causal link between pulsatility and nonsurgical bleeding events in CF-LVADs. ${ }^{4-7}$ Building on this, there is an emerging body of evidence elucidating how the potential mechanisms by which reduced arterial pulsatility and high pump shear stress result in the impairment of peripheral cardiovascular and clotting function. ${ }^{1-4,8}$ Specifically, in vitro studies have documented a shared mechano-transduction pathway in endothelial cells for the secretion of clotting factors and the production of the vasodilator nitric oxide (NO), a key vasodilator that has been recognized to be attenuated in CF-LVAD recipients. ${ }^{9-11}$ Vincent and colleagues ${ }^{1}$ recently revealed a dose-response relationship between reduced pulsatility and attenuated plasma clotting factors in swine implanted with a CF-LVAD. We will briefly review these findings and provide commentary on how CF-LVADs that, by attenuating arterial pulsatility and generating high pump-shear stresses, disrupt clotting factors and appear to result in a "double whammy" for vWF and ultimately

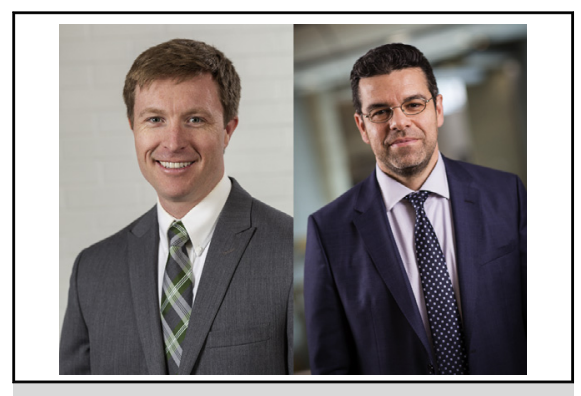

Jay R. Hydren, MS, and Stavros G. Drakos, MD, PhD

Central Message

By attenuating arterial pulsatility and generating high pump shear stresses, CF-LVADs create a "double whammy" effect on vWF, which contributes to nonsurgical bleeding.

The Invited Expert Opinion provides a perspective on this topic based on the following paper: J Amer Coll Card. 2018;19:2106-2118. https:// doi.org/10.1016/j.jacc.2018.02.075.

See Commentary on page 916

nonsurgical bleeding. We will also discuss the potential implications of these phenomena for contemporary heartassist pump technology.

\section{PULSATILITY AND GASTROINTESTINAL BLEEDING: INSIGHT FROM OBSERVATIONAL STUDIES}

Although not always directly measured, attenuated pulsatility has consistently been implicated in nonsurgical bleeding events experienced by CF-LVAD recipients. ${ }^{2}$ Initially, a retrospective cohort study, by our institute, established a strong association between reduced pulsatility and an increased risk of bleeding complications in patients with CF-LVADs. ${ }^{5}$ Pulsatility in this study was evaluated by means of the LVAD parameter pulsatility index and by the echocardiographic assessment of aortic valve opening. Another factor implicated in the occurrence of GIB is acquired vWF syndrome that ensues after CF-LVAD implantation. ${ }^{6,12}$ However, it should be noted that prospective data suggest that vWF deficiency is not solely responsible for the bleeding diathesis observed in CF-LVADs. Crow and colleagues ${ }^{6}$ documented that although a significant reduction of vWF occurred in all 37 
of the CF-LVAD recipients studied, only 10 exhibited GIB. This suggests that there is a compensatory pathway or additional factors involved in the pathophysiology of GIB in this population. A retrospective analysis of 108 LVAD recipients revealed several independent factors associated with GIB, including pulse pressure, age, mean arterial blood pressure, right atrial blood pressure, and previous GIB. ${ }^{7}$ Furthermore, by stratifying this cohort on the basis of a pulse pressure cutoff value of $35 \mathrm{~mm} \mathrm{Hg}$, significant insight into the potential role of pulsatility was provided: Within 9 months of implantation, the high pulsatility group experienced only 8 GIB events ( $83 \%$ unaffected), whereas the low pulsatility group experienced 47 GIB events $(69 \%$ unaffected). In another retrospective study, Patel and colleagues $^{4}$ observed 88 GIB events (75\% unaffected) within 12 months of CF-LVAD support. Of note, in this study, again, the non-GIB group had a higher pulsatility index than the GIB group, and of the 28 patients with GIB who underwent an orthotopic heart transplantation, none had a nonsurgical bleeding event after orthotopic heart transplantation, with a median follow-up time of 2.3 years. Together, these observational studies provide evidence supporting a relationship between reduced arterial pulsatility and the cause of GIB in patients with heart failure who received a CF-LVAD.

It is acknowledged that systemic anticoagulation therapy needed to prevent thrombotic complications within the mechanical support device, which is not well standardized between clinical trial sites and between patients, further complicates the cause of nonsurgical bleeding events. However, in clinical trials looking at the potential effects of removing all anticoagulation therapies, these studies still reveal high bleeding rates, with a $40 \%$ incidence of nonsurgical bleeding in patients who are not receiving anticoagulation therapy. ${ }^{13}$

\section{VON WILLEBRAND FACTOR, NITRIC OXIDE, AND CONTINUOUS-FLOW LEFT VENTRICULAR ASSIST DEVICES: THE ROLE OF PULSATILITY AND PUMP SHEAR}

Both endothelial cells and platelets secrete vWF, with in vitro studies revealing that vWF production in endothelial cells is regulated by a mechano-transduction pathway that shares components that both activate and upregulate $\mathrm{NO} .{ }^{9} \mathrm{NO}$ is the primary dilatory signal for the peripheral vascular function tests, known to be reduced in CF-LVAD recipients compared with age-matched healthy controls and patients with heart failure. ${ }^{8}$ Both vWF and NO share a common intercellular receptor (vascular endothelial growth factor receptor 2), which increases NO through the activation of Akt and the secretion of $\mathrm{vWF}$ into the plasma by the exocytosis of Weibel-Palade bodies through phosphoinositide-specific phospholipase $\gamma 1$ and the calcium pathway. ${ }^{9}$ Pulsatility stimulates vWF production, whereas NO release seems to result in a negative feedback loop, inhibiting the exocytosis of $\mathrm{vWF}$ by endothelial cells (Figure 1,A). Thus, the interwoven nature of NO and vWF has somewhat complicated repercussions in circulatory systems with reduced pulsatility. ${ }^{10,11}$

It seems that CF-LVADs not only decrease vWF production, due to attenuated pulsatility, but also exacerbate degradation by reducing the level of high-molecular-weight von Willebrand factor (HMW vWF), causing diminished vWF-dependent platelet aggregation. ${ }^{2}$ This "double whammy" effect leads to vWF insufficiency and an increased risk of bleeding (Figure 1, A). vWF degradation occurs through 2 known pathways: (1) ventricular assist deviceinduced shear, which activates HMW vWF with a change from a coiled state to a thread-like active state; and (2) ventricular assist device-induced shear, which increases ADAMTS13 activation known to downregulate vWF activity by reducing vWF size at A2 binding domains. ${ }^{14}$ Degradation of vWF through these 2 mechanisms is particularly concerning because physiologic shear needed to activate vWF is only $15(\mathrm{~Pa})$, whereas shear stress is more than 10-fold higher in a CF-LVAD recipient. ${ }^{15}$ Therefore, HMW vWF activation and subsequent degradation are likely to continue to occur, because a greater than a $90 \%$ reduction in pump shear would be needed in newly designed pumps if this were taken into account. The activation of ADAMTS13 is equally concerning because disease states of overactivity, type $2 \mathrm{~A}$ von Willebrand disease, and deficient activity, through genetic and autoimmune processes, result in the loss of clotting factor regulation, causing bleeding disorders or platelet microthrombi. However, promising data from a recent in vitro experiment documented a preservation of HMW $\mathrm{vWF}$ levels with the use of an ADAMTS13 inhibitor (general protease inhibitor ethylenediaminetetraacetic acid), using a mock circulatory loop with the HeartMate II (Abbott, Chicago, Ill) LVAD set at a speed of $9000 \mathrm{rpm} .{ }^{16}$ Together, these mechanisms clearly indicate that the CF-LVAD-induced reduction of HMW vWF levels and subsequent diminished regulatory control of clotting factors will continue to be an issue as long as the pump-induced shear cannot be reduced to physiologic levels.

In combination, these observations describe the multifactorial competing mechanisms that create the considerable challenge of managing the balance between nonsurgical bleeding and thrombotic events in CF-LVAD recipients. Improving our understanding of the nuances associated with the physiologic pathways and mechanisms of action that link vWF, pulsatility, and pump shear to clotting disorders, as well as likely contributors to angiodysplasia such as low-molecular-weight $\mathrm{vWF}$ multimers ${ }^{17}$ and reduced omega- 3 fatty acid intake, ${ }^{18}$ will help advance the efforts to promote the clinical optimization of CF-LVAD therapies. 


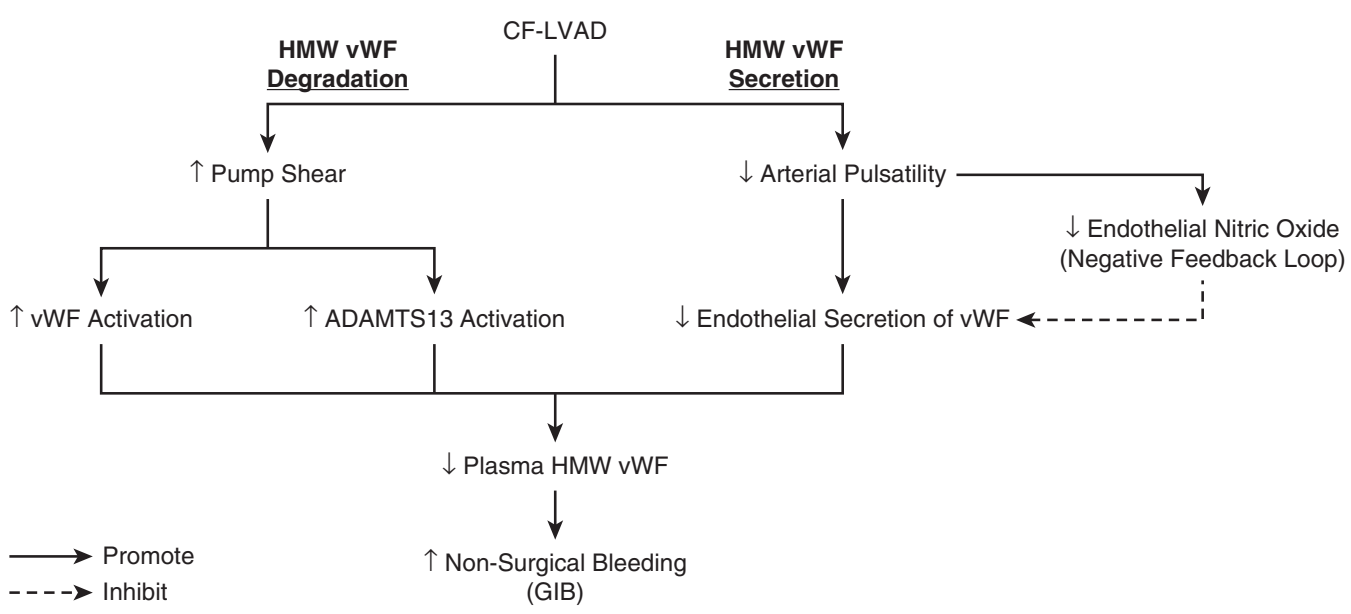

A

\begin{tabular}{ccc} 
Pulsatility & \\
\hline Low & Intermediate & $\begin{array}{c}\text { High } \\
\text { (Physiological) }\end{array}$ \\
\hline
\end{tabular}

\begin{tabular}{|c|c|c|c|c|}
\hline \multirow{2}{*}{\multicolumn{2}{|c|}{$\begin{array}{l}\text { None } \\
\text { (Physiological) }\end{array}$}} & & & \multirow{3}{*}{$\begin{array}{l}\text { HMW vWF: } \\
\text { GIB: } \\
\text { Condition/Model: } \\
\text { Explanted CF-LVAD } 2 \\
\text { Heart Transplant }{ }^{2,4}\end{array}$} \\
\hline & & & & \\
\hline & \multirow{3}{*}{ 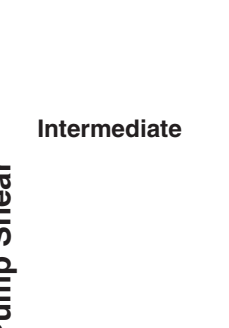 } & & & \\
\hline & & $\begin{array}{l}\text { HMW vWF: } \downarrow \downarrow \\
\text { GIB: } \uparrow \uparrow \uparrow\end{array}$ & $\begin{array}{l}\text { HMW vWF: } \downarrow \downarrow \\
\text { GIB: } \uparrow / \sim\end{array}$ & $\begin{array}{l}\text { HMW vWF: } \\
\text { GIB: }\end{array}$ \\
\hline & & $\begin{array}{l}\text { Condition/Model: } \\
\qquad \mathrm{H}^{17,18}\end{array}$ & $\begin{array}{l}\text { Condition/Model: } \\
\text { EVAHEART } \\
\text { VentrAssist }{ }^{2}\end{array}$ & $\begin{array}{l}\text { Condition/Model: } \\
\text { Human Case Study: } \\
\text { PF-MCS: TAH (CARMAT) }{ }^{1} \\
\text { TAH: CardioWest }^{2}\end{array}$ \\
\hline & High & $\begin{array}{l}\text { HMW vWF: } \downarrow \downarrow \downarrow \\
\text { GIB: } \uparrow \uparrow \uparrow\end{array}$ & HMW vWF: $\downarrow \downarrow$ & HMW vWF: $\downarrow$ \\
\hline \multicolumn{2}{|c|}{$\begin{array}{l}\text { Within Normal Range } \\
\text { Mild Change } \\
\text { Moderate Change } \\
\text { Severe Change }\end{array}$} & $\begin{array}{l}\text { Condition/Model: } \\
\text { Swine CF-LVAD-LV }(A)^{1} \\
\text { Human Case Study } \\
\text { CF-MCS }(E C M O)^{1} \\
\text { CF-MCS+PF-MCS } \\
\text { HMII, HW }{ }^{1}, 4\end{array}$ & $\begin{array}{l}\text { Condition/Model: } \\
\text { Swine CF-LVAD-LV (B) }{ }^{1}\end{array}$ & $\begin{array}{l}\text { Condition/Model: } \\
\text { Swine CF-LVAD-Ao (B) }{ }^{1}\end{array}$ \\
\hline
\end{tabular}

B

FIGURE 1. A, The 2 physiologic pathways by which CF-LVADs result in a reduction in HMW vWF multimers. The exacerbated degradation and attenuated endothelial secretion of HMW vWF lead to nonsurgical bleeding events, such as GIB. B, The results from observations in patients or models in which both arterial pulsatility and pump shear are reasonably well documented with the corresponding observed change in HMW vWF and the effect on the incidence of GIB. Swine experiments by Vincent and colleagues ${ }^{1}$ documented a dose-related response of HMW vWF by varying arterial pulsatility with similar pump shear using 2 different pumps (Models A and B) and 2 different pump placements: left ventricle and aorta. In a human case study reported by Vincent and colleagues, ${ }^{1}$ a patient in cardiogenic shock underwent continuous-flow mechanical circulatory support, pulsatile flow mechanical circulatory support, and a combination of both (mechanical circulatory support + pulsatile flow mechanical circulatory support), facilitating the assessment of the subsequent changes in HMW vWF in the context of arterial pulsatility and pump shear. To provide further information regarding the potential interaction between reduced arterial pulsatility and pump shear, studies looking specifically at vWF or GIB in patients with heart failure implanted with a mechanical circulatory support system were included: LVAD pumps: HeartMate II (Abbott, Chicago, Ill); HeartMate3, HeartWare HVAD (HeartWare Inc, Framingham, Mass), EVAHEART (EVAHEART Inc, Houston, Tex); and 2 total artificial heart devices: CardioWest (SynCardia Systems, Tucson, Ariz) and CARMAT (Vélizy-Villacoublay, France). CF-LVAD, Continuous-flow left ventricular assist device; $H M W v W F$, high-molecular-weight von Willebrand factor; GIB, gastrointestinal bleeding. 
PULSATILITY AND PUMP SHEAR INTERACTIONS ON VON WILLEBRAND FACTOR: FURTHER INSIGHT AND IMPLICATIONS

Recent work has confirmed the hypothesis that arterial pulsatility, even in the presence of high pump-induced shear, can positively influence plasma levels of HMW vWF. Specifically, in a pair of swine studies, Vincent and colleagues ${ }^{1}$ documented a causal relationship between arterial pulsatility and HMW vWF while keeping pumpinduced shear constant. In the first component of the study, the authors demonstrated a dose-response relationship between reduced arterial pulse pressure and the loss of HMW vWF, which was observed in only 30 minutes. Perhaps even more persuasive is the second component of the study that used a cross-over design to manipulate pulsatility in a swine model, which documented that HMW vWF loss was attenuated with normal pulsatile conditions compared with low pulsatile conditions after only 2 hours of pump support (high shear), signifying that vWF secretion into the plasma was likely elevated by the greater pulsatility. The recovery of HMW vWF and increased vWF antigen plasma levels occurred within only 60 minutes, between trials, when only the native heart contributed to blood flow, suggestive of increased secretion vWF when endothelial cells were reexposed to normal pulsatility. In an effort to provide some translational data, a unique case report of a patient in cardiogenic shock revealed a reduction in HMW vWF after initial support with continuous-flow venoarterial extracorporeal membrane oxygenation; after only 3 days with a pulsatile total artificial heart (CARMAT, Vélizy-Villacoublay, France), HMW vWF levels recovered. However, 7 days later, the same patient was put on co-support (ie, total artificial heart and continuous-flow venoarterial extracorporeal membrane oxygenation, resulting in loss of arterial pulsatility and increased pump shear), the previous recovery of HMW vWF was lost. Ideally, future studies aimed at evaluating the effect of high and low arterial pulsatility and pump shear should include data from all 4 possible combinations of conditions, as depicted in Figure 1, B. Unfortunately, because of the type of mechanical pumps tested, low shear and low pulsatility in the swine and human experiments were not possible, and in the human case study a high-shear, high-pulsatility condition was also not attainable (Figure 1, $B)$. These missing conditions limit the ability to determine the independent and interaction effects of arterial pulsatility and pump shear on vWF plasma levels. Regardless, these dose-response and translational data further demonstrate the potential for a causal link between reduced arterial pulsatility and CF-LVAD pump shear on plasma HMW vWF levels.

\section{POTENTIAL TO IMPROVE BLEEDING OUTCOMES THROUGH PUMP DESIGN}

A better understanding of the interaction between reduced arterial pulsatility and pump shear has been elusive because of the limited availability of device shear rates and reporting of arterial pulsatility. ${ }^{2}$ In an effort to illuminate this critical interaction, Figure $1, B$ provides a summary of the magnitude of HMW vWF loss and GIB events at various combinations of arterial pulsatility and pump shear levels, with reference to supporting evidence. By examining the 2 extremes, (1) CF-LVAD, high shear and low pulsatility, and (2) natural heart, no shear and normal pulsatility, Heilmann and colleagues ${ }^{2}$ reported HMW vWF loss and subsequently acquired von Willebrand syndrome in 9 patients with heart failure receiving the HeartMate II or VentrAssist (Ventracor, Chatswood, Australia). With pump explantation or heart transplantation, all these patients recovered. In a third condition, with a total artificial heart (CardioWest; SynCardia Systems, Tucson, Ariz), elevated shear, and normal pulsatility, 3 patients exhibited neither a decrease in HMW vWF nor nonsurgical bleeding complications after 94 to 191 days of support. ${ }^{2}$ These data begin to provide evidence suggesting that the optimization of arterial pulsatility and pump shear could be achievable, which could decrease the nonsurgical bleeding side effect of circulatory assist pumps.

The HeartMate 3 device is recognized to provide artificial pulsatility, likely in an effort to acknowledge the importance of this basic circulatory phenomenon. However, because the HeartMate 3 pump algorithm was designed to primarily "flush out" potential clot formations in the pump, rather than provide sufficient artificial arterial pulsatility, the results from the Momentum 3 clinical trial are not surprising: The HeartMate 3 device eliminated the incidence of pump thrombosis (HeartMate II: $12.2 \%$, HeartMate 3: $0.0 \%$ ); however, there was no change in GIB (HeartMate II: $27.3 \%$ vs HeartMate $327.0 \%$ ). ${ }^{19}$ With regard to HMW vWF, Netuka and colleagues ${ }^{20}$ documented a decrease of approximately $18 \%$ with the HeartMate 3 device compared with a $46 \%$ to $73 \%$ reduction, depending on the analytic technique used, with the HeartMate II device after 45 days of support, with no measurable differences in ADAMTS13 activity levels or functional assays of vWF between pumps. This suggests that the HeartMate 3 device may still induce a mechanical shear stress adequate to disturb vWF homeostasis. Given the reviewed evidence suggesting a causal relationship among arterial pulsatility, the secretion of HMW vWF, and GIB, it seems that the HeartMate 3 device likely provides an arterial pulsatility below the physiologic minimum level needed to reduce nonsurgical bleeding events. Our group recently investigated the impact of pump speed modifications on pulsatility (using HeartWare [HeartWare Inc, Framingham, Mass] and HeartMate II pumps). We found that with relatively small rpm changes, well within the usual clinical range of rpm, we were able to induce an increase in brachial artery pulsatility index of up to approximately $38 \%$, demonstrating that optimizing 
pump speed to manipulate peripheral pulsatility in the outpatient setting is feasible. ${ }^{2}$

The EVAHEART (EVAHEART Inc, Houston, Tex) is a pump designed to reduce HMW vWF degradation through a series of features to increase pulsatility and reduce pump shear: reduced operating rpm range, larger flow gaps between impeller and housing, magnetically levitated centrifuge (shear $<1500 \mathrm{~N} / \mathrm{m}^{2}$ ), and a flat $\mathrm{H}-\mathrm{Q}$ curve, which can provide a pulse pressure of 15 to $30 \mathrm{~mm} \mathrm{Hg}{ }^{3}$ Compared with the HeartMate II, the EVAHEART has been reported to induce a smaller increase in the smallest vWF fragments (1.7-fold vs 5.5-fold) in a mock circulatory loop with whole human blood. ${ }^{22}$ It was presumed that these fragments are degradations of $\mathrm{HWM} \mathrm{vWF}^{22}$ and this was later confirmed in a human trial. ${ }^{23}$ Of note, in a postmarket approval study in Japan, none of the 93 patients receiving the EVAHEART had GIB on follow-up and no pumps were exchanged for pump thrombosis. ${ }^{3}$ Although limited, these data are encouraging and provide evidence that a multifactorial effort to increase arterial pulsatility and reduce pump shear have promise to diminish the clinically observed incidence of nonsurgical bleeding in LVAD recipients.

As new pump designs work to eliminate major side effects in the order of critical importance and increase the breadth of potential recipients, we encourage and look forward to pump designs aimed at mitigating nonsurgical bleeding and thrombosis. This could include pulsatility algorithms to mimic the physiologic pulsatile shear stress of a normal heartbeat and approaches to drastically reduce mechanical shear known to activate clotting factors.

\section{CONCLUSIONS}

Evidence points at CF-LVAD causing a "double whammy" effect on vWF by both increasing the rate of degradation and decreasing secretion into the plasma, leading to an increase in bleeding. Although recent studies have provided promising advances in the working hypothesis for the mechanism responsible for the bleeding complications exhibited by CF-LVAD recipients, we still need more answers with respect to the potential to minimize pump-induced shear and the level of arterial pulsatility required to abate bleeding events.

\section{Conflict of Interest Statement}

S.G.D. is on the advisory board for Abbott and receives personal payment for consulting. All other authors have nothing to disclose with regard to commercial support.

\section{References}

1. Vincent F, Rauch A, Loobuyck V, Robin E, Nix C, Vincentelli A, et al. Arterial pulsatility and circulating von Willebrand factor in patients on mechanical circulatory support. J Am Coll Cardiol. 2018;71:2106-18.
2. Heilmann C, Geisen U, Beyersdorf F, Nakamura L, Benk C, Berchtold-Herz M, et al. Acquired von Willebrand syndrome in patients with ventricular assist device or total artificial heart. Thromb Haemost. 2010;103:962-7.

3. Saito S, Yamazaki K, Nishinaka T, Ichihara Y, Ono M, Kyo S, et al. Post-approval study of a highly pulsed, low-shear-rate, continuous-flow, left ventricular assist device, EVAHEART: a Japanese multicenter study using J-MACS. J Heart Lung Transplant. 2014;33:599-608.

4. Patel SR, Oh KT, Ogriki T, Sims D, Shin JJ, Madan S, et al. Cessation of continuous flow left ventricular assist device-related gastrointestinal bleeding after heart transplantation. ASAIO J. 2018;64:191-5.

5. Wever-Pinzon O, Selzman CH, Drakos SG, Saidi A, Stoddard GJ, Gilbert EM, et al. Pulsatility and the risk of nonsurgical bleeding in patients supported with the continuous-flow left ventricular assist device HeartMate II. Circ Heart Fail. 2013;6:517-26.

6. Sheri C, Chen D, Milano C, Thomas W, Joyce L, Piacentino V III, et al. Acquired von Willebrand syndrome in continuous-flow ventricular assist device recipients. Ann Thorac Surg. 2010;90:1263-9; discussion 1269.

7. Joly JM, El-Dabh A, Kirklin JK, Marshell R, Smith MG, Acharya D, et al. High right atrial pressure and low pulse pressure predict gastrointestinal bleeding in patients with left ventricular assist device. J Card Fail. 2018;24: 487-93.

8. Witman MAH, Garten RS, Gifford JR, Groot HJ, Trinity JD, Stehlik J, et al. Further peripheral vascular dysfunction in heart failure patients with a continuous-flow left ventricular assist device: the role of pulsatility. JACC Heart Fail. 2015;3:703-11.

9. Xiong Y, Hu Z, Han X, Jiang B, Zhang R, Zhang X, et al. Hypertensive stretch regulates endothelial exocytosis of Weibel-Palade bodies through VEGF receptor 2 signaling pathways. Cell Res. 2013;23:820-34.

10. Lanzarone E, Gelmini F, Fumero A, Carini M, Costantino ML, Fumero R, Alfieri O. Preservation of endothelium nitric oxide release during beating heart surgery with respect to continuous flow cardiopulmonary bypass. Perfusion. 2010;25:57-64.

11. Lanzarone E, Gelmini F, Tessari M, Menon T, Suzuki H, Carini M, et al. Preservation of endothelium nitric oxide release by pulsatile flow cardiopulmonary bypass when compared with continuous flow. Artif Organs. 2009;33: 926-34.

12. Uriel N, Pak SW, Jorde UP, Jude B, Susen S, Vincentelli A, et al. Acquired von Willebrand syndrome after continuous-flow mechanical device support contributes to a high prevalence of bleeding during long-term support and at the time of transplantation. J Am Coll Cardiol. 2010;56:1207-13.

13. Katz JN, Adamson RM, John R, Tatooles A, Sundareswaran K, Kallel F, et al. Safety of reduced anti-thrombotic strategies in HeartMate II patients: a oneyear analysis of the US-TRACE Study. J Heart Lung Transplant. 2015;34: 1542-8.

14. Proudfoot AG, Davidson SJ, Strueber M. von Willebrand factor disruption and continuous-flow circulatory devices. J Heart Lung Transplant. 2017;36: 1155-63.

15. Nascimbene A, Neelamegham S, Frazier OH, Moake JL, Dong JF. Acquired von Willebrand syndrome associated with left ventricular assist device. Blood. 2016; $127: 3133-41$

16. Van Belle E, Rauch A, Vincentelli A, Jeanpierre E, Legendre P, Juthier F, et al. Von Willebrand factor as a biological sensor of blood flow to monitor percutaneous aortic valve interventions. Circ Res. 2015;116:1193-201.

17. Bartoli CR, Zhang DM, Hennessy-Strahs S, Kang J, Restle DJ, Bermudez C, et al. Clinical and in vitro evidence that left ventricular assist device-induced von Willebrand factor degradation alters angiogenesis. Circ Heart Fail. 2018; 11:e004638.

18. Imamura T, Nguyen A, Rodgers D, Kim G, Raikhelkar J, Sarswat N, et al. Omega-3 therapy is associated with reduced gastrointestinal bleeding in patients with continuous-flow left ventricular assist device. Circ Heart Fail. 2018;11: e005082.

19. Fried J, Sayer G, Naka Y, Uriel N. State of the art review: evolution and ongoing challenges of left ventricular assist device therapy. Structural Heart. 2018;1-12.

20. Netuka I, Kvasnička T, Kvasnička J, Hrachovinová I, Ivák P, Mareček F, et al. Evaluation of von Willebrand factor with a fully magnetically levitated centrifugal continuous-flow left ventricular assist device in advanced heart failure. $J$ Heart Lung Transplant. 2016;35:860-7.

21. Hydren JR, Kithas AC, Park SH, Wever-Pinzon O, Selzman CH, Perry WH, et al Peripheral vascular pulsatility in heart failure patients with continuous flow centrifuge and axial left ventricular assist devices: the effect of pump speed. ASAI J. April 4, 2019 [Epub ahead of print]. 
22. Bartoli CR, Kang J, Zhang D, Howard J, Acker M, Atluri P, Motomura T. Left ventricular assist device design reduces von Willebrand factor degradation: a comparative study between the HeartMate II and the EVAHEART left ventricular assist system. Ann Thorac Surg. 2017;103:1239-44.
23. Ichihara Y, Nishinaka T, Komagamine M, Yamada Y, Miyamoto T, Suzuki K et al. Gastrointestinal bleeding was rare with centrifugal type continuous flow left ventricular assist device EVAHEART. J Heart Lung Transplant. 2013;32: S233-4. 